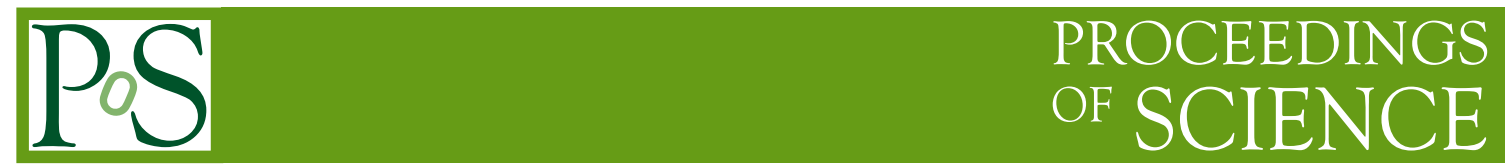

\title{
Light meson spectroscopy at BESIII
}

\author{
Francesca De Mori*' \\ Università di Torino and INFN sez. Torino \\ E-mail: demori@to.infn.it
}

The BESIII Experiment at the Beijing Electron Positron Collider (BEPC II) has collected large data samples in the tau-charm mass region. Among them, BESIII has the world's largest samples of $J / \psi$ and $\psi(3686)$ that are an ideal and clean laboratory to study light hadron spectroscopy and search for exotics. Recent BESIII results in this field are presented in this contribution

The European Physical Society Conference on High Energy Physics

5-12 July, 2017

Venice

* Speaker.

${ }^{\dagger}$ On behalf of BESIII collaboration 


\section{Introduction}

Light hadron spectroscopy (LHS) is a unique laboratory to verify Quantum Chromo Dynamics (QCD) in low energy regime, where the perturbative approach is not holding anymore. Beside the conventional hadrons predicted by quark model, QCD allows also exotic hadrons like glueballs, multiquark states molecules and hybrids. One of the main aims of LHS is the search for exotic states. Many candidates have been reported, but none of them has been unambiguously established yet.

The Beijing Electron Spectrometer III (BESIII) detector [1] is a general purpose magnetic spectrometer, installed at the Beijing Electron Positron Collider II (BEPCII). BEPCII is a double ring $e^{+} e^{-}$collider, hosted at the Institute of High Energy Physics (IHEP) of Beijing (P. R. C.) with a tunable beam energy from 1 to $2.3 \mathrm{GeV}$.It has recently reached the design luminosity of $10^{33} \mathrm{~cm}^{-2} \mathrm{~s}^{-1}$. BESIII has collected the world's largest samples of $J / \psi$ and $\psi(3686)$ since the beginning of its successfully operation in 2008. The world's largest data samples of $J / \psi$ and $\psi(2 S)$ have been collected by BESIII. In $20121.09 \times 10^{9} \mathrm{~J} / \psi$ and $3.41 \times 10^{8} \psi(2 S)$ were collected to be added to the 2009 data samples of $2.25 \times 10^{8} J / \psi$ and $1.06 \times 10^{8} \psi(2 S) . J / \psi$ and $\psi(2 S)$ radiative decays are a clean and powerful environment to study light hadron spectroscopy.

\section{Enhancement at $p \bar{p}$ threshold}

A strong enhancement at $p \bar{p}$ threshold, called $\mathrm{X}(p \bar{p})$, was observed for the first time by BESII [2] in $J / \psi \rightarrow \gamma p \bar{p}$, being confirmed later by BESIII [3] and CLEO-c [4] in the same channel with the $J / \psi$ coming from $\psi(2 S) \rightarrow \pi^{+} \pi^{-} J / \psi$. Analogous structures have not been observed at $p \bar{p}$ threshold in related channels as radiative $\psi(2 S)$ decays, $\Upsilon(1 S) \rightarrow \gamma p \bar{p}$ or $J / \psi \rightarrow \omega p \bar{p}$ [5]. The nature of $\mathrm{X}(p \bar{p})$ has stimulated many interpretations. Among them, a very appealing one explains it as a $p \bar{p}$ bound state, the so-called baryonium [6]. Based on 2009 data, a Partial Wave Analysis of $J / \psi \rightarrow \gamma p \bar{p}$ has been performed, including Final State Interaction, below $2.2 \mathrm{GeV} / c^{2}$ [7]. The spinparity quantum numbers of the structure were found to be $0^{-+}$with a statistical significance larger than $30 \sigma$. The mass and width for this state were found to be $1832_{-5}^{+19}(\text { stat })_{-17}^{+18}($ syst $) \pm 19$ (model) $\mathrm{MeV} / \mathrm{c}^{2}$ and $13 \pm 39(\text { stat })_{-13}^{+10}($ syst $) \pm 4($ model $\left.)\right) \mathrm{MeV} / \mathrm{c}^{2}\left(\Gamma<76 \mathrm{MeV} / \mathrm{c}^{2}\right.$ at the $90 \%$ confidence level), respectively.

In the same mass-region BESII observed a structure in $\eta^{\prime} \pi^{+} \pi^{-}$invariant mass in the $J / \psi \rightarrow$ $\gamma \eta^{\prime} \pi^{+} \pi^{-}$decays [8], named X(1835). It has been confirmed by BESIII [9] in the same process, with mass and width equal to $1836.5 \pm 3.0_{-2.1}^{+5.6} \mathrm{MeV} / c^{2}$ and $190 \pm 9_{-36}^{+38} \mathrm{MeV} / c^{2}$. Its angular distribution has been found to be consistent with $J^{P}=0^{-}$. Its nature is not yet fully understood and several hypothesis have arose about that, as a $p \bar{p}$ bound state or a glueball candidate.

Using $1.09 \times 10^{9} \mathrm{~J} / \psi$ collected in 2012 , BESIII was able to study the $\eta^{\prime} \pi^{+} \pi^{-}$invariant mass to investigate the coupling to $p \bar{p}$. An abrupt in the line-shape has been found near the $p \bar{p}$ mass threshold [10]. Two models have been used to characterize the distorted line-shape near the threshold with a similar agreement with the data. The first one uses the Flatté formula [11] and incorporates the opening of the $p \bar{p}$ decay channel in the mass spectrum. A large coupling of $\mathrm{X}(1835)$ to $p \bar{p}$ is found with significance larger than $7 \sigma$ and an additional resonance, named X("1920"), is needed with mass and width of $1918.6 \pm 3.0 \mathrm{MeV} / c^{2}$ and $50.6 \pm 20.9 \mathrm{MeV} / c^{2}$, respectively. The fit result 

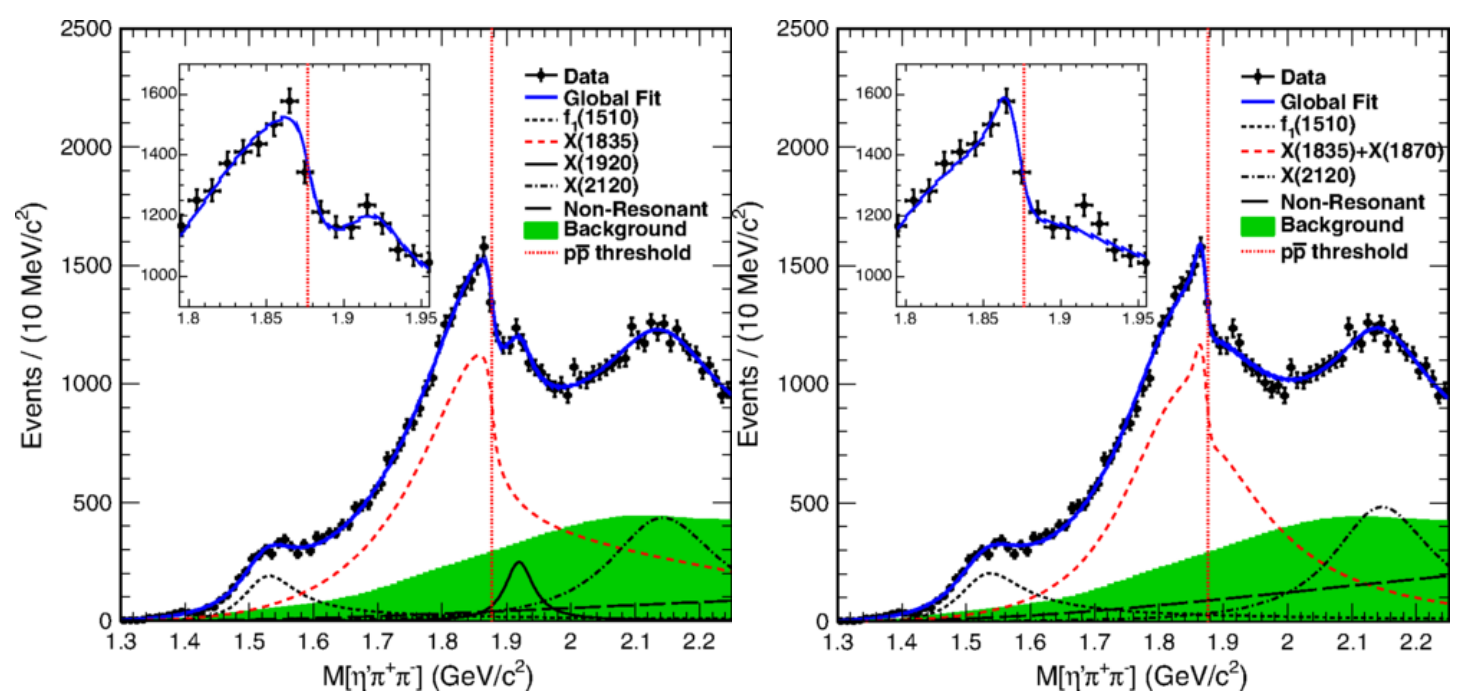

Figure 1: The fit results are shown: on the left Flattee formula is applied while on the right the coherent sum of two Breit Wiegner amplitudes is used [10].

is shown in Fig. 1 on the left. It suggest most likely the existence of a broad state above the $p \bar{p}$ threshold that can be explained as a $p \bar{p}$ molecule-like state because of the strong coupling to $p \bar{p}$. The second model consists in the coherent sum of two resonant amplitudes. It is assumed that the distortion of the line-shape is due to the existence of a narrow resonance near the $p \bar{p}$ threshold and to the interference between $\mathrm{X}(1835)$ and this resonance.The fit results for the second model are shown in Fig 1 on the right. The $\mathrm{X}(1835)$ mass results to be $1825.3 \pm 2.4 \mathrm{MeV} / c^{2}$ with a width of $245.2 \pm 13.1 \mathrm{MeV} / c^{2}$, while the $\mathrm{X}(1870)$ mass and width are $1870.2 \pm 2.2 \mathrm{MeV} / c^{2}$ and $13.0 \pm 6.1 \mathrm{MeV} / c^{2}$, with a statistical significance of $7.9 \sigma . \mathrm{X}$ ("1920") turns out to be not significant in this case. Also in this case a state is found, but narrow and below the $p \bar{p}$ threshold. It can be explained most likely as an unconventional meson, i.e. the $p \bar{p}$ bound state.

More investigations and more data are needed to clarify the nature of the states around 1.85 $\mathrm{GeV} / c^{2}$.

Among the other studies it is important to search for other decay modes. The $\mathrm{X}(1835)$ was also observed in $J / \psi \rightarrow \gamma \eta K_{S}^{0} K_{S}^{0}$ decays based on $1.3 \times 10^{9} \mathrm{~J} / \psi$ events, that represent the full statistics of 2009 and 2012. Being similar to the one in which it was discovered this channel is favourable. Furthermore, due to exchange symmetry and $\mathrm{CP}$ conservation, the two main background channels are forbidden: $J / \psi \rightarrow \eta \pi^{0} K_{S}^{0} K_{S}^{0}$ and $J / \psi \rightarrow \eta K_{S}^{0} K_{S}^{0}$. The remaining background is well known and minimal uncertainties arise from its model. A clear structure has been observed in $\eta K_{S}^{0} K_{S}^{0}$ mass spectrum, around $1.85 \mathrm{GeV} / c^{2}$, strongly correlated to $f_{0}(980)$. A Partial Wave Analysis has been performed for $M\left(K_{S}^{0} K_{S}^{0}\right)<1.1 \mathrm{GeV} / \mathrm{c}^{2}$ and $M\left(K_{S}^{0} K_{S}^{0} \eta\right)<2.8 \mathrm{GeV} / \mathrm{c}^{2}$ and the results are shown in Fig. 2 on the left. PWA includes a X(1835) resonance in the $f_{0}(980) \eta$ and non-resonant component in one of the possible decay channels $f_{0}(980) \eta, f_{0}(1500) \eta$ or $f_{2}(1525) \eta$. In the nominal solution the $\mathrm{X}(1835)$ is required with a significance larger than $12.9 \sigma$ and the mass and width have been determined to be $\left(1844 \pm 9_{-25}^{+16}\right) \mathrm{MeV} / c^{2}$ and $\left(192_{-17-43}^{+20}\right) \mathrm{MeV} / c^{2}$, respectively. The spin-parity of $\mathrm{X}(1835)$ turns out to be $J^{P}=0^{+-}$[12]. The resonance parameters are consistent with those obtained for X(1835) from $J / \psi \rightarrow \gamma \eta^{\prime} \pi^{+} \pi^{-}$by BESIII, while this structure results to be wider 

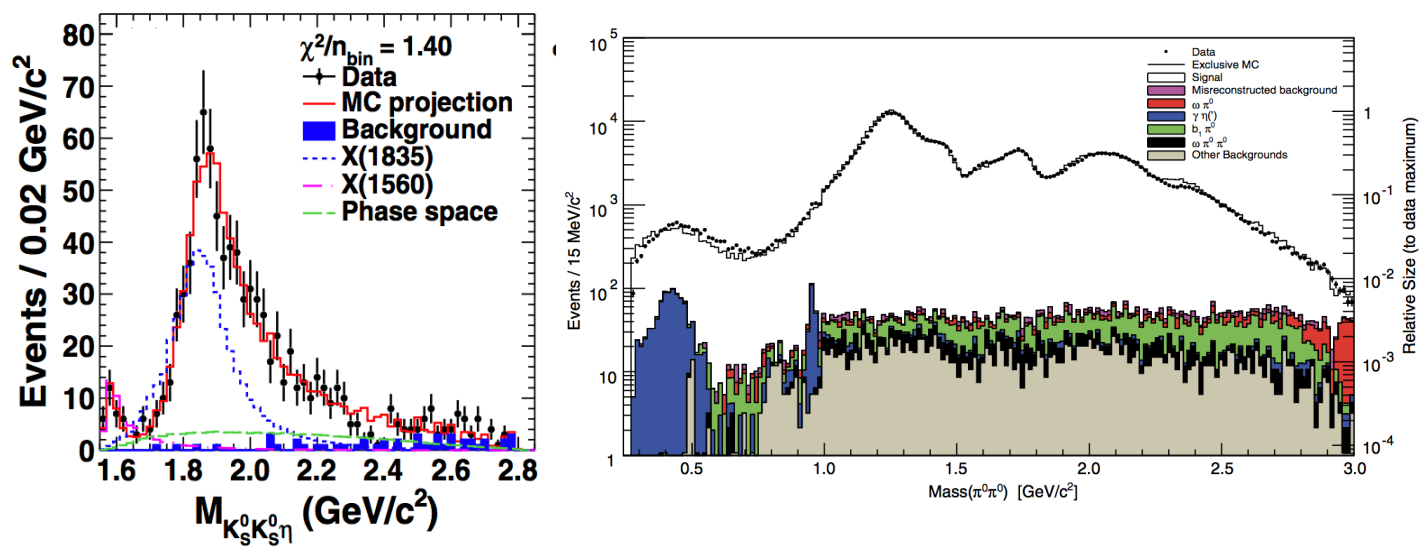

Figure 2: On the left, the comparisons between data and PWA fit projections is shown for the $K_{s} K_{s} \eta$ invariant mass distribution (two entries/event) [12]. On the right, it is shown the $\pi^{0} \pi^{0}$ invariant mass spectrum, in logarithmic scale, with coloured histograms of the background contributions [13].

than the $\mathrm{X}(p \bar{p})$. Whether the $\mathrm{X}(p \bar{p})$ and $\mathrm{X}(1835)$ are the same state and which is their nature are still open questions. In any case a strong coupling to $p \bar{p}$ has been found for both these states.

\section{Selected results on Partial Wave Analysis of Charmonia decays}

In this section few selected results of Partial Wave Analysis of Charmonia will be highlighted. In spite of the success of Standard Model, the connection between QCD and hadron dynamics is still requiring a full understanding. Despite the strong effort in experimental investigations, the low mass isoscalar scalar meson spectrum, for example, remains poorly understood. One of the reasons is the presence of broad overlapping resonances and the opening of thresholds. The light scalar spectrum is very interesting, in particular because the lightest glueball is foreseen to be a scalar. The investigation of this spectrum,by means of radiative $J / \psi$ decays, can provide complementary information with respect to the hadronic production and BESIII can profit of the aforementioned large data samples in giving a crucial contribution.

Based on $1.3 \times 10^{9} \mathrm{~J} / \psi$ events, that represent the full statistics of 2009 and 2012, a Model Independent PWA (MIPWA) of $J / \psi \rightarrow \gamma \pi^{0} \pi^{0}$ has been performed [13]. The study of two pseudoscalar spectrum in radiative $J / \psi$ decay profits of the simplicity of the amplitude analysis. In addition, for the chosen neutral channel, sizable challenging backgrounds, like $\rho \pi$, are not present.

In MIPWA, the components of the $\pi \pi$ amplitude are measured independently for many small intervals in the $\pi \pi$ invariant mass: a piecewise complex function can be built to describe the sdependence of $\pi \pi$ interaction. The aim is to provide the description of scalar and tensor components of $\pi^{0} \pi^{0}$ system, minimizing the systematic bias due to the assumptions on $\pi \pi$ dynamics.

Clear structures were observed with a background level of $1.8 \%$. The MIPWA results indicate that the scalar contributions are mainly from $\sigma(600), f_{0}(1370), f_{0}(1500), f_{0}(1710)$ and $f_{0}(2020)$, while tensor component is dominated from $f_{2}(1270)$.

This model independent description can be integrated with other results from complementary reactions for their use in phenomenological models. Finally, the branching fraction of this radiative decay is measured to be $(1.15 \pm 0.05) \times 10^{-3}$, where the error is systematic only and the statistical 

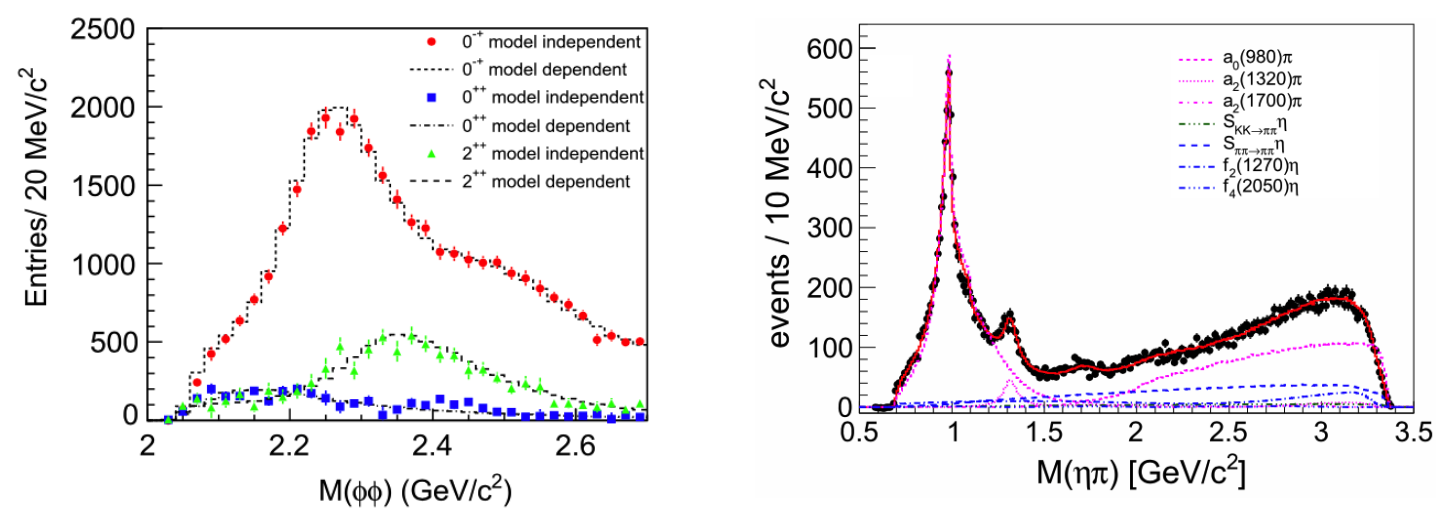

Figure 3: On the left the intensities of the individual $J^{P C}$ components obtained from MIPWA and model dependent PWA are shown for comparison purposes [14]. On the right, projection of data in $\eta \pi$ invariant mass together with our base-line fit (solid curve) and the corresponding amplitudes (dashed and dotted lines description can be found in the legend) [15].

error is negligible.

Furthermore a PWA of the decay $J / \psi \rightarrow \gamma \phi \phi$ has been performed to investigate the intermediate states [14]. The results have shown that the structures are dominated by $0^{+}$states. The $\eta(2225)$ existence has been confirmed and two additional pseudoscalars have been observed: $\eta(2100)$ and $\mathrm{X}(2500)$. In the meanwhile we have observed the scalar $f_{0}(2100)$ and three tensor states, $f_{2}(2010)$, $f_{2}(2300)$ and $f_{2}(2340)$. The large production rate measured for this last state make it a good tensor glueball candidate, being consistent with Lattice QCD predictions. Using this data sample both MIPWA and model dependent PWA have been performed with well consistent results, as shown in Fig. 3 on the left.

The last selected result is the most recent one and it profits of the world's largest data sample of about $35000 \chi_{c 1}$ from $\psi(3686)$ radiative decay, that is more than 15 times CLEO-c data sample. The amplitude analysis has been performed on $\psi(3686) \rightarrow \gamma \chi_{c 1} ; \chi_{c 1} \rightarrow \eta \pi^{+} \pi^{-}$, aiming to search for exotic mesons with $J^{P C}=1^{-+}$decaying in $\eta \pi$ in the (1.4-2.0) GeV/ $/ c^{2}$ mass region [15]. The results of the PWA are shown in Fig. 3, on the right, together with the projection in the $\eta \pi$ invariant mass. The dominant two-body structure observed is $a_{0}(980)^{ \pm} \pi^{\mp}$ with $a_{0}(980)$ decaying in $\eta \pi$ and a significant non-zero coupling of $a_{0}(980)$ to the $\eta^{\prime} \pi$, that turns out to be important to describe the $a_{0}(980) \rightarrow \eta \pi$ distribution. Regarding the search of exotic mesons, upper limits for the production of the $\pi_{1}(1400) \pi, \pi_{1}(1600) \pi$ and $\pi_{1}(2015) \pi$ have been determined. The $a_{2}(1700) \pi$ production in $\chi_{c 1} \rightarrow \eta \pi^{+} \pi^{-}$decays is observed for the first time with a significance larger than $17 \sigma$ and mass and width in agreement with world average values, providing a further evidence of its existence.

\section{References}

[1] M. Ablikim et al. (BESIII Collaboration), Nucl. Instrum. Meth. A 614, 345 (2010)

[2] J. Z. Bai et al. (BESII Collaboration), Phys. Rev. Lett. 91, 022001 (2003)

[3] M. Ablikim et al. (BESIII Collaboration), Chin. Phys. C 34, 421 (2010) 
[4] J. P. Alexander et al. (CLEO Collaboration), Phys. Rev. D 82, 092002 (2010)

[5] M. Ablikim et al. (BESIII Collaboration), Eur. Phys. J. C 53, 15 (2008);

[6] A. Datta and P. J. ODonnel, Phys. Lett. B 567, 273 (2003); M. L. Yan et al., Phys. Rev. D 72, 034027 (2005); B. Loiseau and S. Wycech, Phys. Rev. C 72, 011001 (2005)

[7] M. Ablikim et al. (BESIII Collaboration), Phys. Rev. Lett. 108, 112003 (2012)

[8] J. Z. Bai et al. (BESII Collaboration), Phys. Rev. Lett. 95,262001 (2005);

[9] M. Ablikim et al. (BESIII Collaboration), Phys. Rev. Lett. 106,072002 (2011);

[10] M. Ablikim et al. (BESIII Collaboration), Phys. Rev. Lett. 117, 042002 (2016);

[11] S. M. Flatté, Phys. Lett. B 63,224 (1976);

[12] M. Ablikim et al. (BESIII Collaboration), Phys. Rev. Lett. 115, 091803 (2015);

[13] M. Ablikim et al. (BESIII Collaboration), Phys. Rev. D 92, 052003 (2015);

[14] M. Ablikim et al. (BESIII Collaboration), Phys. Rev. D 93, 112011 (2016);

[15] M. Ablikim et al. (BESIII Collaboration), Phys. Rev. D 95, 032002 (2017); 